\title{
L'équilibre des Pouvoirs et la Répartition des Compétences en Matière de Conclusion des Traités dans les Etats d'Afrique Francophone. Du rendez- vous Manqué à la Rencontre Fertile
}

\author{
Oumarou Sanda, \\ Doctorant en droit public, Moniteur à la Faculté des Sciences Juridique \\ Politique de l'Université de Ngaoundere, Cameroun
}

URL:http://dx.doi.org/10.19044/esj.2019.v15n32p1

\section{Résumé}

L'internationalisation du droit constitutionnel est une évidence que les Etats d'Afrique francophone ne sauraient ignorer. La distribution des «compétences constitutionnelles internationales » entre les pouvoirs, qui constitue une des manifestations de l'internationalisation, n'est pas sans incidence sur leur équilibre. Les analyses doctrinales de cette répartition des prérogatives entre les organes des pouvoirs exécutif et législatif ont, dans leur majorité, forgé l'idée d'une prééminence du Président de la République dans la conclusion des traités et accords internationaux. Ce déséquilibre affirmé par la doctrine, à l'examen des Constitutions africaines, remet au goût du jour le respect des exigences élémentaires de la séparation des pouvoirs ; et mérite, de ce fait, l'attention de la présente réflexion. Celle-ci a pour objet de relativiser cette solution de principe, mécaniquement transposée aux régimes constitutionnels de conclusion des engagements internationaux des Etats d'Afrique subsaharienne, et revêt ainsi un double intérêt: théorique et pratique. Pour y parvenir, l'étude comparée des différents systèmes constitutionnels des Etats africains d'expression française à la lumière du positivisme normativiste, et à certains égards sociologique, a permis de constater un certain déséquilibre dans l'attribution et la délimitation de la compétence de négociation des conventions internationales. Cependant, l'articulation des compétences de ratification/approbation constitue le point, par excellence, d'équilibre entre les pouvoirs exécutif et législatif dans la procédure de conclusion des traités. Dans cet esprit, le chef de l'Etat ne saurait plus être regardé comme le seul maître à bord dans l'expression du consentement définitif à être lié ; le Parlement ne se trouve pas en retrait.

Mots clés: Traité/accord international, Compétence, Négociation, Ratification, Approbation, Afrique francophone 


\title{
The Balance of Powers and the Distribution of Jurisdictions in Concluding Treaties in Francophone African States. From the Missed Appointment to the Fertile Encounter
}

\author{
Oumarou Sanda, \\ Doctorant en droit public, Moniteur à la Faculté des Sciences Juridique \\ Politique de l'Université de Ngaoundere, Cameroun
}

\begin{abstract}
The internationalization of constitutional law is obvious that Frenchspeaking African states cannot ignore. The distribution of "international constitutional jurisdiction" among the powers, which is one of the manifestations of internationalization, is not without impact on the balance relating thereto. The doctrinal analyses of this distribution of prerogatives between the organs of the executive and the legislative powers have, for the most part, forged the idea of a pre-eminence of the President of the Republic in the conclusion of international treaties and agreements. This imbalance affirmed by the doctrine, on the examination of the African Constitutions, brings up to date the respect of elementary requirements of the separation of the powers, thus the rationale of the present thought. It is about relativizing this purely provisional solution, transposed mechanically to the constitutional regimes of conclusion of the international commitments of Sub-Saharan Africa States, and thus has a double interest: theoretical and practical. To achieve this, the comparative study of the different constitutional systems of French-speaking African States with the means of normativist and, in some respects, sociological positivism, has allowed us to note a certain imbalance in the attribution and delimitation of the scope of jurisdiction in the negotiation of international conventions. However, the articulation of the powers of ratification/approval constitutes the point, par excellence, of balance between the executive and legislative powers in the procedure of conclusion of treaties. The head of State cannot be regarded as the only master on board in the expression of the final consent to be bound; the Parliament does not remain in the background.
\end{abstract}

Keywords: Treaty/international Agreement, Jurisdiction, Negotiation, Ratification, Approval. French speaking Africa 


\section{Introduction}

La répartition des compétences relatives à la conclusion des traités internationaux est susceptible de bouleverser «les grands équilibres à l'intérieur de l'Etat» (Tourard, 2000, p. 12). De la modalité du partage des compétences, en matière de conclusion des traités dépend, en effet, la préservation, ou alors l'altération, des attributions classiques internes, des pouvoirs exécutif et législatif: l'équilibre entre eux. Cette distribution des prérogatives, au visa de la séparation des pouvoirs (Barberis, 2012, p. 717), entre l'exécutif et le législatif constitue, dans ce sens, un terrain fertile au déséquilibre. Sous ce rapport, il devient impérieux, de rechercher, de trouver, mais surtout d'appliquer une formule permettant de préserver les attributions traditionnelles respectives des pouvoirs exécutif et législatif à chacune des phases de la procédure de formation des traités, normes par excellence du droit international (Roussillon, 2000, p. 180). Dans cette perspective, la tâche n'est pas mince.

A travers la prévision des dispositions relatives aux conventions internationales (Kombi, 2003, p. 8 ; Kontchou, 1979, p. 234), faisant références (Tall, 2017, p. 123), ou plus simplement qui renvoient aux aspects du droit international (Sall, 1997, p. 340 ; Tcheuwa, 1999, p. 96), les Etats d'Afrique subsaharienne se sont ouvertement refusés de rester à l'écart de ce mouvement d'internationalisation des Constitutions (Ondoua, 2014, p. 439). Les textes fondamentaux de ces Etats dédient, pour la plupart, un titre entier aux traités et accords internationaux : du régime de leur formation extérieure à celui de leur incorporation, en passant par l'indication de leur place dans la hiérarchie normative nationale. Le premier qui seul constitue l'objet de la présente réflexion, est aménagé dans ces Etats d'Afrique francophone, avec des similitudes qui ne sauraient cependant masquer les traits spécifiques de chaque Constitution.

Les constituants des «Etats africains de succession française » (de Gaudusson, 2008, p. 675), et « belge » consacrent, dans leur grande majorité, à quelques différences près, des régimes de conclusion des traités internationaux, associant successivement les pouvoirs exécutif et législatif. Cette configuration transparait clairement dans les Constitutions du Benin (article 144), du Burkina-Faso (article 148), du Cameroun (article 43), de la République de Côte d'Ivoire (article 119), de la République Gabonaise (article 113), du Mali (article 114), du Niger (article 168), de la Guinée Conakry (article 77), de la République Centrafricaine (article 91), de la République du Congo (article 218), de la République Démocratique du Congo (article 213), du Sénégal (article 95), du Tchad (article 219). Ces énonciations constitutionnelles à incidence internationales (Kombi, 2003, p. 8), réalisent suivant des formulations diverses, un melting-pot des organes relevant des 
pouvoirs exécutif et législatif dans la phase normative des relations extérieures : la confection des traités internationaux.

Les différences, plus ou moins légères, observables entre les Constitutions de ces anciennes colonies françaises ou belges, quant à la lettre de leurs prescriptions, ne sauraient dissimuler leur trame de fond commune : l'implication combinée des organes de l'exécutif et du législatif dans la procédure de conclusion des engagements internationaux. Ici comme dans d'autres domaines, semble bien avoir joué " la loi d'imitation » de la Constitution française de la Ve République (Gonidec, 1982, p. 297 ; Tall, 2017, p. 123). Cette incantation de l'esprit de la Constitution de 1958 (article 52 et suivants) dans les Constitutions des Etats d'Afrique francophone n'a pas laissé la doctrine insensible.

La question de la répartition des «compétences constitutionnelles internationales »(Guetzevich, 1936, p. 220) en matière de conclusion des traités dans les Constitutions des Etats africains francophones n'est, en effet, pas nouvelle. Si jusqu'à une certaine période, les études relatives à cette question étaient rares (Gonidec, 1982, p. 296) tel n'est plus l'état de la réalité aujourd'hui. Faisant mécaniquement écho aux idées libérales (Locke, 1953, p. 158 et Montesquieu, 1964, p. 586) qui confiaient la conduite des relations internationales et la conclusion des traités en particulier au pouvoir exécutif, la doctrine majoritaire aboutit à la conclusion de la prépondérance (Troper, 1998, p. 403 ; Bugorgue-Larsen, 2009, p. 1310) ou de la prééminence (Zoller, 1992, p. 31 ; Ascensio, 2012, p. 679) du Président de la République (Tcheuwa, 1999, p. 97 ; Kombi, 2003, pp. 10-16 ; Ondoua, 2017, p. 163). Cette thèse majoritaire a du poids ; elle n'est pas infaillible pour autant.

Deux arguments complémentaires pourraient, à titre principal et liminaire, être avancés en faveur d'une relativisation de cette position de principe. Premièrement, cette conception majoritaire semble ignorer les subtilités, sources de spécificité, ou mieux de richesse du régime constitutionnel de chaque Etat, et partant du nouveau constitutionnalisme en Afrique (Kanté, 2011, pp. 240-247). Bien plus, et c'est le second argument, cette affirmation généralisée paraît minorer la répartition interne des compétences, postulée par le principe fort de la séparation des pouvoirs. Cellelà semble surtout « (...) noyer la conclusion des traités internationaux dans l'étendue plus vaste de l'activité diplomatique »(Goesel-Le Bihan, 1995, p. 25), alors même que la première ne saurait, en droit strict, être ensevelie sous le voile des mystères de la seconde.

Cet empressement doctrinal à reproduire, dans un contexte purement africain ces conclusions libérales laisse une brèche dans l'appréhension exacte de la logique de la distribution des pouvoirs en matière de conclusion des normes conventionnelles. Il semble, dans cet ordre d'idées, qu'il y'ait de la 
place pour une étude consacrée à la conclusion des traités internationaux sous le prisme, cette fois ci, de l'équilibre entre les pouvoirs exécutif et législatif.

La présente réflexion se propose ainsi, non pas d'infirmer totalement la thèse de la primauté de l'exécutif dans la procédure de conclusion des traités, seulement de la relativiser à partir d'une problématique différente et simple : les Constitutions des Etats d'Afrique francophone réalisent-elles un équilibre des pouvoirs en matière de conclusion des traités ? Plus exactement, ces dispositions constitutionnelles à incidence internationale prennent-elles en compte la distribution interne des attributions entre les organes de l'exécutif et du législatif, afin d'en préserver la substance dans la conduite des relations extérieures ? Ces interrogations soulèvent, en toile de fond, la question de la coïncidence féconde ou de la distance stérile entre l'équilibre nécessaire des organes exécutif et législatif d'un côté et la répartition constitutionnelle des attributions relatives à la conclusion des traités internationaux d'un autre côté. Le premier (l'équilibre) et la seconde (la répartition des compétences) se rencontrent-ils en fin de compte dans les Etats d'Afrique noire francophone ?

Un tel diagnostic impose que l'on s'accorde d'abord sur l'entendement de l'objet étudié. Le traité international désigne un « accord international conclu par écrit entre Etats et régi par le droit international, qu'il soit consigné dans un document unique ou dans deux ou plusieurs instruments connexes, et quelle que soit sa dénomination particulière » (article 2, alinéa 1, lettre (a) de la Convention de Vienne du 23 mai 1969, sur le droit des traités). C'est « l'expression de volonté concordantes, émanant de sujet de droit dotés de la capacité requise, en vue de produire des effets juridiques régis par le droit international » (Pellet, et Miron, 2015, p. 293). Seront par conséquent exclu de l'étude, les engagements internationaux non pris en compte par les Constitutions des Etats d'Afrique francophone : les gentlemen's agreement, les actes concertés non conventionnels, les actes unilatéraux etc. La compétence est entendue comme l' « aptitude juridique à prendre des actes correspondant à l'exercice d'une fonction » (De Villers, et Le Divellec, 2017, p. 62). Seuls les deux axes forts du processus d'élaboration et d'adoption des traités que constituent la négociation et la ratification/approbation (Goesel LeBihan, 1995, p. 29), seront pris en compte par la présente investigation. La négociation sera entendue comme l' « échange de vues et de documents, entre des personnes habilitées à représenter un Etat ou une organisation internationale en vue d'établir en commun le contenu d'un acte conventionnel » (Salmon, 2001, p. 735), tandis que la ratification renverra à 1'« acte par lequel l'autorité étatique la plus haute, détenant la compétence constitutionnelle de conclure les traités internationaux, confirme le traité élaboré par ses plénipotentiaires, consent à ce qu'il devienne définitif et obligatoire et s'engage solennellement au nom de l'Etat à l'exécuter » (Daillier, Forteau et Pellet, 2009, p. 153) . L'équilibre des pouvoirs est, quant 
à lui, appréhendé comme la stabilité, ou la coïncidence, entre les compétences juridiques internes reconnues à chacun des organes des pouvoirs exécutif et législatif et celles qui leur sont dévolues dans le cadre de la confection des normes internationales conventionnelles.

L'objet de l'étude apparaît ainsi intéressant à un double titre. D'une part il s'agit d'une question, qui parce que placée à la charnière des ordres juridiques interne et international (Simon, 1995, p. IX), est d'une actualité constante et mérite de ce fait une attention renouvelée. D'autre part, cette réflexion permet de vérifier, ou mieux d'éprouver, la pertinence de l'affirmation suivant laquelle en matière internationale les Parlements africains constituent des « coquilles vides » (Desouches, 1979, p. 96) qui ne servent «à rien» (Sindjoun, 1993, p. 813) : des vassaux obéissant des exécutifs dominants et surpuissants (Vandendriessche, 2001, p. 59).

L'examen des dispositions constitutionnelles réalisant la répartition des compétences entre les pouvoirs, suivant les méthodes positiviste normativiste et sociologique, autorise à parler d'un déséquilibre au niveau de la compétence de négocier et, à l'inverse, d'un équilibre dans la phase essentielle visant à exprimer le consentement définitif à être lié : le moment de ratifier, ou d'approuver les traités internationaux. Plus exactement, si la négociation constitue le site d'un certain déséquilibre des pouvoirs (I), l'articulation de la procédure de ratification/approbation, par contre, constitue le siège d'un équilibre certain (II) entre ces organes de l'exécutif et du législatif.

\section{Un certain déséquilibre dans la distribution de la compétence de négociation des traités internationaux}

Si la négociation des traités internationaux est, «par nature, une matière régie par le droit international, elle relève aussi nécessairement du droit interne »(Daillier, Forteau et Pellet, 2009, p. 141). Celle-là se trouve donc sous l'emprise de deux ordres juridiques : le droit international et le droit interne (Kombi, 1992, p. 163). Les Constitutions, normes suprêmes des ordres juridiques, des Etats d'Afrique francophone réalisent, au profit du Chef de l'Etat, un certain déséquilibre dans l'organisation des compétences en matière de négociation des traités internationaux. L'attribution du pouvoir de négociation révèle une inégalité dévoilée entre les pouvoirs exécutif et législatif (A), à rebours de sa délimitation qui trahit une inégalité déguisée des organes du pouvoir exécutif (B).

\section{A. L'inégalité dévoilée des pouvoirs exécutif et législatif, l'attribution de la compétence de négociation.}

L'attribution des compétences en matière de négociations des engagements internationaux constitue l'axe majeur du déséquilibre dans la 
répartition des compétences constitutionnelles internationales entre le Parlement et les organes du pouvoir exécutif. Les constituants des Etats d'Afrique francophone dévoilent, en effet, clairement ce déséquilibre, ou mieux cette inégalité, en excluant le pouvoir législatif de la phase des négociations (1). A l'identique, ces Constitutions admettent exclusivement le pouvoir exécutif (2) à conduire des négociations visant la conclusion des traités.

\section{L'exclusion du pouvoir législatif}

Dans l'ordre juridique international, la négociation a des fonctions multiples : elle « permet de cerner les intentions des parties, d'ajuster leur vue unilatérale, de concilier les intérêts parfois opposés et souvent parfois divergents »(Basdevant, 1963, p. 409). Elle «consiste en un débat, une discussion entre les représentants d'intérêts contraires, discussion dans laquelle chacun présente ses raisons et conteste celles des autres » (Amougou, 2006, p. 1519). Si le but des négociations est donc «celui de parvenir à un accord entre participants qui, en général, se matérialise dans un traité » (Geamanu, 1980, p. 432), alors on « imagine mal que le corps législatif conduise lui-même les négociations internationales » (Troper, 1998, p. 140) dans les matières qui ressortissent du domaine de la loi. Le Parlement paraît ainsi frappé d'une inaptitude congénitale à initier et conduire efficacement la négociation d'une convention internationale.

L'exacte ampleur de cette infirmité parlementaire à négocier les traités internationaux est consacrée de manière générique par les Constitutions des Etats d'Afrique noire francophone. C'est en réalité le Président de la République qui seul négocie « (...) les engagements internationaux » (article 77 de la Constitution de la Guinée Conakry ; article 95 de la Constitution du Sénégal), « (...) les traités et accords internationaux » (article 148 de la Constitution du Burkina-Faso ; article 43 de la Constitution du Cameroun, article 119 de la Constitution de Côte d'Ivoire, article 144 de la Constitution du Benin, article 168 de la Constitution du Niger, article 213 de la Constitution de la République Démocratique du Congo). L'exclusion africaine du pouvoir législatif, à travers cette tenue à distance des chambres, base et haute, de la négociation des Conventions internationales n'est pas démentie par les Constitutions du Congo et du Gabon (article 218 paragraphe II et article 113).

Les Etats d'Afrique francophone s'accordent ainsi, non sans pertinence, à marquer une inégalité entre les pouvoirs exécutif et législatif au niveau de la négociation du contenu des normes conventionnelles. L'exclusion constitutionnelle du Parlement est commandée par l'admission à titre exclusif des organes du pouvoir exécutif. 


\section{L'admission exclusive du pouvoir exécutif}

Les Etats africains de succession française ou belge qui semblent bien unanimes quant au principe de l'exclusion du Parlement de la conduite de toute négociation visant à la conclusion d'un traité international s'accordent également à ne confier, à titre exclusif, cette compétence qu'aux organes du pouvoir exécutif. Cependant, il existe une légère divergence dans l'indication de l'organe autorisé à effectuer les négociations internationales. Il convient, dans cette logique de distinguer les Constitutions qui accordent cette prérogative de négocier exclusivement à la personne du Chef de l'Etat, de celles qui la partagent entre celui-ci et le Gouvernement.

L'option constitutionnelle d'attribution du pouvoir de négocier à la seule personne du Président de la République ne va pas sans susciter quelques inquiétudes. En précisant que "le Président de la République négocie (...) » (les articles 148 de la Constitution du Burkina-Faso ; 43 de la Constitution du Cameroun ; 119 de la Constitution de Côte d'Ivoire, 144 de la Constitution du Benin ; 168 de la Constitution du Niger ; 213 de la Constitution de la République Démocratique de Congo), cette disposition s'avère non seulement d'une interprétation peu aisée, mais surtout elle est susceptible d'engendrer des blocages dans l'exercice de la politique extérieure, et partant de la diplomatie.

Une interprétation littérale ou linguistique (Ost et De Kerchove, p. 50. cités par Goesel-Le Bihan, 1995, p. 21) de cette disposition peut donner à penser que c'est le Président de la République qui doit, « lui-même » (Manin, 1979, p. 667) négocier les traités. A l'inverse, l'interprétation systématique, qui a la préférence de cette étude, en donne un sens fort différent. Suivant celle-ci, en effet, cette disposition constitutionnelle abrite une " norme permissive » (Zoller, 1992, p. 218), et remplit plus exactement une fonction d'habilitation au profit du Président de la République. La méthode systématique constitue ainsi une bouée de sauvetage dans l'articulation des attributions du Chef de l'Etat en matière de négociation. En commandant, en effet, de ne plus considérer le texte constitutionnel « comme une juxtaposition des dispositions régissant des domaines distincts » (Goesel-Le Bihan, 1995, p. 27), mais comme un ensemble de normes entretenant des liens de complémentarité, cette méthode permet d'éviter tout blocage dans la conduite des relations extérieures, plus exactement dans l'exercice des compétences diplomatiques.

A l'inverse, certaines Constitutions africaines partagent cette compétence de négociation, suivant la nature de l'engagement international en cause, entre le Président de la République et le Gouvernement. En prévoyant, à l'image de la Constitution française de 1958 révisée, que : «Le Président de la République négocie (...) les traités », et est seulement " informé de toute négociation tendant à la conclusion d'un accord international ", les 
Constitutions du Congo (article 218 paragraphe II), du Gabon (article 113), de la République de Côte d'ivoire (article 119 alinéa 2) et du Tchad (article 219), reconnaissent expressément un rôle d'initiative international au Gouvernement. Bien plus, elles réaffirment leur exclusion du Parlement de la phase des négociations visant à la conclusion d'une convention internationale. Cette tenue à l'écart du pouvoir législatif de la phase des pourparlers ne saurait pour autant inférer un équilibre entre le Chef de l'Etat et le Gouvernement dans la répartition de la compétence de négociation des engagements internationaux. En réalité cette marque d'inégalité entre le premier et le second, cache mal, ou mieux déguise, une inégalité entre ces « deux planètes »(Olinga, 2013, p. 42) du pouvoir exécutif dans la délimitation de la compétence de négocier.

\section{B. L'inégalité déguisée des organes du pouvoir exécutif, la délimitation de la compétence de négociation.}

L'équilibre volontairement manqué dans l'attribution de la compétence de négocier ne semble pas parfaitement retrouvé dans sa délimitation. Le Président de la République et le Gouvernement ne sont pas mis à pied d'égalité dans la distribution de la compétence de négociation. Si la simplicité du critère de délimitation des compétences (1), de l'un par rapport à celles de l'autre, pourrait conduire à le penser, il n'en est rien à la vérité. A l'examen, ce critère de délimitation des compétences de négociation du Gouvernement et du Président de la République paraît brouillé (2).

\section{Un critère a priori simple de délimitation des compétences de négociation, la dichotomie traité/accord.}

Le critère de délimitation des compétences du Président de la République et du Gouvernement en matière de négociation des engagements est de prime abord simple. Les articles 218 de la Constitution du Congo, 119 alinéa 2 de la Côte d'ivoire, 219 de la Constitution du Tchad, et 113 de la Constitution de la République gabonaise, pendant en Afrique francophone de l'article 52 de la Constitution française révisée, consacrent en effet, comme critère de détermination des compétences de négociations des engagements conventionnels, la dichotomie traité-accord. C'est du moins ce qui semble ressortir de la lecture de ces dispositions constitutionnelles : « Le Président de la République négocie (...) les traités », et il est seulement «informé de toute négociation tendant à la conclusion d'un accord international ».

La dénomination de l'engagement international à négocier se révèle donc être l'axe principal de démarcation de la compétence du Chef de l'Erat par rapport à celle du Gouvernement. Et inversement la qualification constitutionnelle, traité ou accord, dudit engagement international pourrait découler de la seule identification de l'auteur de sa négociation. Constitue, 
dans ce sens, un accord «l'instrument qui est négocié par le ministre des affaires étrangères, ou en son nom, et qui fait l'objet, le cas échéant, d'une approbation» (Isidoro, 2009, p. 1299). Par contre constitue un traité, « l'instrument qui est négocié par le Président de la République, ou en son nom, et ratifié par lui » (Gaia, 1991, p. 8). Vu sous cet angle, cette dichotomie paraît à la fois pertinente et simple. Le besoin d'assurer l'unité et la cohérence de la politique extérieure (Kombi, 1996, p. 19) justifie bien que le Chef de l'Etat soit informé de toute négociation tendant à la conclusion d'un accord non soumis à ratification. Une telle exigence semble davantage commandée par la fonction de garant du respect des traités dévolue au Président de la République (article 5 de la Constitution du Cameroun ; article 41 alinéa 2 de la Constitution du Togo).

La simplicité du critère tient, quant à elle, à l'équilibre qu'il prétend établir entre les deux piliers du pouvoir exécutif. Il tend surtout à ne pas laisser en retrait, le premier Ministre et son Gouvernement, en leur confiant seulement les seconds rôles, dans les différentes phases du déroulement des négociations internationales (Ondoua, 2017, p. 163). Ce critère ouvre une alternative simple : les accords ressortissent de la compétence de négociations du Gouvernement ; les traités relèvent du portefeuille présidentiel.

En tirant les conséquences de l'attribution de la compétence de ratification au Président de la République, cette dichotomie reconnaît au Gouvernement la compétence résiduelle de négocier exclusivement les accords situés hors de cette orbite : « accord non soumis à ratification ». Chacun des deux géants du pouvoir exécutif dispose donc d'une part importante du bargaining power (pouvoir de négociation). L'objectif constitutionnel de réalisation d'un équilibre entre le Président de la République et le Gouvernement semble, dans cette perspective, et seulement dans celle-ci, bien atteint. Toutefois, à l'analyse, cette dichotomie aux apparences simples se révèle fort défectueuse (Le Bœuf, 2016, p. 607) et brouille par conséquent la délimitation des compétences de négociation au sein du pouvoir exécutif.

\section{Une délimitation brouillée de la compétence de négociation du Président de la République et du Gouvernement.}

La simplicité de la formule constitutionnelle suivant laquelle « Le Président de la République négocie (...) les traités», et est seulement «informé de toute négociation tendant à la conclusion d'un accord international », ne saurait occulter la difficulté de son maniement. A l'épreuve de la pratique, cette dichotomie "traité négocié par le Chef de l'Etat' et "'accord négocié par le Gouvernement', se révèle inopérante. Le caractère peu formaliste de l'ordre juridique international d'un côté et la complexité matérielle de distinguer le domaine du traité de celui de l'accord d'un autre 
côté, alimentent et entretiennent un brouillard, favorable au Chef de l'Etat, dans la délimitation de la compétence de négocier des deux têtes du pouvoir exécutif.

La systématicité apparente de la distinction «négociation des traités par le Président de la République » et «négociation des accords par d'autres autorités », est tout d'abord fragile (Le Bœuf, 2016, p. 604) et semble de ce fait artificielle. Au vu des deux conventions de Vienne sur le droit des traités (23 mai1969 et 21 mars 1986), qui n'attachent aucune importance à cette distinction, l'on est fondé à douter de sa valeur pratique, du moins d'un point de vue international. L'article 2 commun à ces deux textes laisse, en effet, une grande marge de discrétion aux Etats parties dans le choix de la dénomination de leur engagement international. Mieux encore, ces Conventions de Vienne sont silencieuses quant aux domaines respectifs des différentes dénominations possibles.

Bien moins, les procédures solennelle et simplifiée que semble impliquer respectivement la distinction "traités" et "accords" ne se déterminent pas a priori en fonction d'un objet ou d'un contenu particulier des normes conventionnelles à négocier. Dans cet élan, il a été affirmé qu' « il n'existe aucun critère rationnel présidant à la répartition des matières entre engagements en forme solennelle et accords en forme simplifiée. Les deux procédures peuvent être employées indifféremment » (Melin-Soucramanien et Pactet, 2014, p. 574). Plus précisément, ce sont les circonstances internationales qui, plus que les considérations de droit interne, déterminent la procédure courte ou longue qui sera suivie pour la formation des traités. Dans cette perspective, la dichotomie entre la négociation présidentielle des traités d'un côté, et celle gouvernementale des accords d'un autre côté, « est à la fois quelque peu superfétatoire et théorique » (Manin, 1979, p. 669). On peine alors à se convaincre de la pertinence de ce critère de répartition pour assurer une certaine égalité, ou même un équilibre, entre le Gouvernement et le Président de la République.

L'absence d'un domaine spécifiquement consacré à la conclusion d'un traité ou d'un accord brouille en pratique la délimitation des compétences entre ces deux géants du pouvoir exécutif. Le Chef de l'Etat pourrait ainsi ventiler de manière fort discrétionnaire, voire arbitraire, la répartition des compétences de négociation au sein du pouvoir exécutif, dont il est par ailleurs le chef. La pratique des Etats d'Afrique francophone paraît bien correspondre avec cette montée en puissance de celui-là au détriment du Gouvernement. Ces Constitutions africaines qui organisent une égalité de façade entre les « deux planètes » de l'exécutif, déguisent en réalité une inégalité au profit du Président de la République en ne spécifiant pas clairement les domaines respectifs des accords et traités internationaux. 
Ce rendez-vous manqué de l'équilibre des pouvoirs tant au niveau de l'attribution, qu'à celui de la délimitation de la compétence de négocier, les engagements internationaux dans les Etats d'Afrique francophone, ne saurait suffire à inférer que toute «la conduite des relations internationales est l'apanage du Chef de l'Etat » (Verhoeven, 1980, p. 250). Celui-ci n'est, en réalité, plus le « seul maitre à bord » au moment de la ratification/approbation des traités internationaux dont l'articulation réalise un équilibre certain entre les pouvoirs.

\section{Un équilibre certain dans l'articulation de la compétence de ratification des traités internationaux.}

S'il est une compétence dont l'articulation constitutionnelle, dans les Etats d'Afrique francophone, révèle une égalité entre les pouvoirs exécutif et législatif, la ratification/approbation est bien celle-là. Les Constitutions africaines sont imprégnées du souci de démocratiser la conduite des relations internationales (De Visscher, 1952, p. 535), et la ratification des traités en particulier. Généralement «perçue comme relevant de la haute politique » (Cabanis et Martin, 2010, pp. 81-82), la ratification constitue, à l'examen, le point d'équilibre entre les pouvoirs exécutif et législatif au regard de la parfaite projection des compétences respectives du premier et du second, qui y est effectuée (A). La nécessité de protéger cet équilibre trouvé au niveau de la ratification explique la consécration du contrôle juridictionnel de l'observance des compétences respectives des pouvoirs exécutif et législatif (B) dans la procédure de conclusion des traités.

\section{A. Un équilibre trouvé, la projection des compétences des pouvoirs exécutif et législatif dans l'organisation de la ratification.}

Que la compétence législative du Parlement doive être protégée dans l'ordre international semble aujourd'hui s'imposer avec la force de l'évidence (Goesel-Le Bihan, 1995, p. 72). Tout serait, en effet, perdu si les compétences internes reconnues au Parlement en matière législative s'effaçaient devant les prérogatives de ratification ou d'approbation, du Président de la République et du Gouvernement. Le besoin de conserver « le monopole de la production normative » (Metou, 2009, p. 132), tout en le rendant compatible avec le principe de la séparation des pouvoirs, semble justifier chez les constituants africains l'adoption d'une précaution : la réalisation d'une symétrie entre les compétences internes et internationales de chacun des organes étatiques. La ratification apparaît dans cette perspective, non seulement comme un moyen de préservation des compétences traditionnelles des pouvoirs exécutif et législatif (1), mais davantage comme un moment d'expression de l'interdépendance de l'exercice des compétences des pouvoirs exécutif et législatif (2) dans la conduite des relations extérieures. 


\section{La ratification, un moyen de préservation des domaines de compétences traditionnelles des pouvoirs exécutif et législatif.}

Prises au pied de la lettre, les Constitutions des Etats d'Afrique francophone assurent, suivant des formulations présentant des légères différences, la préservation des attributions internes des pouvoirs législatif et exécutif : le domaine matériel de la loi (a) et le domaine règlementaire (b).

\section{a. La préservation du domaine matériel de la loi}

Les Parlements des Etats d'Afrique francophone ont leur mot à dire dans la procédure de ratification et/ou d'approbation des engagements internationaux, suivant le contenu et la nature de l'engagement en cause. Plus exactement, en présence d'un traité ou d'un accord international relatif à certaines matières précises, l'autorisation parlementaire constitue un passage obligé pour sa ratification ou son approbation. Les dispositions relatives à la ratification étant parmi celles « qui changent le moins », (Sall, 1997, p. 31), les Constitutions du Bénin (article 145), du Burkina-Faso (article 149), de la République Centrafricaine (article 69), de la République du Congo (article 178), de la République de Côte d'ivoire (article 120), de la République Démocratique du Congo (article 214), de la Guinée Conakry (article 149), du Mali (article 115), du Niger (article 169), du Sénégal (article 77), du Tchad (article 220), du Togo (article 139), imposent une autorisation parlementaire, comme condition substantielle à la ratification d'une catégorie d'engagements internationaux.

Il s’agit, de manière générique de ceux relatifs à la paix, au commerce, à l'organisation internationale, de ceux qui engagent les finances de l'Etat, ceux qui sont relatifs à l'état des personnes, qui comportent cession ou adjonction de territoire, mais surtout de ceux qui modifient les dispositions de nature législative,. Ce dernier «chef d'intervention de l'autorisation parlementaire » (Simon, 1995, p. IX), visant à préserver le domaine matériel de la loi pourrait être regardé comme le dénominateur commun des Constitutions africaines en matière de ratification/approbation. En faisant mention du «domaine de la loi tel que défini à l'article 26 (...) », la loi constitutionnelle camerounaise du 18 janvier 1996 (article 43), ne se trouve pas en retrait de cette logique de préservation des compétences législatives, face à cette « (...) avancée du droit international dans l'ordre juridique interne » (Tourard, 2000, p. 171).

Pour tout traité ou accord international touchant à l'une des matières qui ressortissent du domaine de la loi, l'approbation et/ou la ratification dudit texte, par le Président de la République ou par le Gouvernement, est subordonnée à une autorisation en forme législative du Parlement. Le cas de 
la Constitution camerounaise (article 43), qui attribue la compétence d'approbation au Parlement mérite attention.

En effet, à travers la précision suivant laquelle «Les traités et accords internationaux qui concernent le domaine de la loi, défini à l'article 26 cidessus, sont soumis, avant ratification à l'approbation en forme législative », le constituant camerounais semble amalgamer l'approbation, compétence généralement reconnue à l'exécutif, et l'autorisation de ratification, qui constitue le moyen de sauvegarde des compétences parlementaires (Saidj, 1979, p. 50). A l'examen, ce « flottement terminologique » (Basdevant, 1926, p. 577) du constituant de 1996 ne saurait réellement embarrasser le juriste. Il s'agit à cet article 43 du texte constitutionnel camerounais d'une attribution expresse de la compétence d'autorisation de ratification au Parlement, en cohérence avec l'absence de la dichotomie traité soumis à ratification et accord soumis à approbation (Voir supra).

Au-delà des légers décalages constitutionnels dans l'énumération des matières requérant l'autorisation parlementaire préalable, les Etats d'Afrique francophone semblent tous animés par le souci d'éviter une amputation des compétences normatives parlementaires sous le couvert de la conduite des relations internationales. Le pouvoir exécutif ne peut ainsi faire par la voie de la conclusion d'une convention internationale, ce qu'il ne peut réaliser dans le droit interne sans le concours du Parlement (Duguit, 1924, p. 790 ; Kazadi, 2013, p. 135). La préservation du domaine réglementaire est justiciable des mêmes remarques.

\section{b. La préservation du domaine règlementaire}

Le domaine règlementaire désigne, de manière négative, l'ensemble des matières dans lesquelles le pouvoir exécutif peut intervenir de manière autonome par l'édiction des règlements autonomes (Chapus, 2001, p. 68). Ce domaine est prévu par les articles 28 de la Constitution camerounaise, 100 de la Constitution du Benin, 126 de la Constitution de la République du Congo, 51 de la Constitution du Gabon, 128 de la Constitution de la République Démocratique du Congo en des termes voisins : «Les matières autres que celles qui sont du domaine de la loi ont un caractère réglementaire » ou alors « ressortissent au pouvoir réglementaire ».

La préservation du domaine matériel de la loi, des incursions du pouvoir exécutif, réalisée par les Constitutions africaines n'est pas sans incidence sur la sauvegarde du domaine réglementaire. L'évitement des débordements normatifs, tels qu'établi par la dichotomie domaine réglementaire/ domaine de la loi, constitue le pivot de répartition des compétences constitutionnelles entre les pouvoirs exécutif et législatif dans la phase de la ratification/approbation des traités internationaux. L'ensemble des matières sur lesquelles le Président de la République ou le Gouvernement est 
constitutionnellement habilité à édicter des règlements autonomes restent soustraites du domaine d'exercice de l'autorisation législative. Les traités et ou accords internationaux portant sur le domaine règlementaire ne nécessitent, par conséquent, pas l'assentiment du Parlement avant leur ratification. En ces matières, les organes du pouvoir exécutif conservent leur marge de discrétion dans toute son étendue. S'il n'est cependant pas exclu que ces derniers puissent soumettre lesdits accords ou traités internationaux à l'appréciation du Parlement, afin de leur conférer une couche supplémentaire de légitimité, il convient de relever qu'il s'agit moins d'une exigence constitutionnelle que d'un simple « (...) moyen technique de faire pénétrer les idées démocratiques internes dans la communauté internationale »(Guetzevich, 1936, p. 295). Le pouvoir exécutif reste ainsi maître de toute la procédure de ratification des traités et des accords internationaux portant sur les matières relevant du domaine règlementaire.

En conséquence de la ratification/approbation des traités et accords en rapport avec le domaine matériel de la loi, qui nécessitent une autorisation parlementaire préalable, celle des conventions internationales touchant au domaine réglementaire est tributaire de la seule volonté du pouvoir exécutif. Ces deux pouvoirs sont ainsi aptes à protéger leurs domaines de compétences respectifs au moment de la ratification ou de l'approbation des engagements internationaux. Il s'agit d'une des manifestations les plus éclatantes de l'équilibre entre l'exécutif et le législatif, résultante de « (...) l'applicabilité de la séparation des pouvoirs à la procédure de conclusion des traités » (Goesel Le-Bihan, 1995, p. 29). Un tel mécanisme de préservation des champs de compétences des organes des pouvoirs exécutif et législatif engendre inévitablement une interdépendance entre ces derniers au moment de la ratification/approbation des traités et/ou accords internationaux en rapport avec le domaine matériel de la loi.

\section{La ratification, un moment d'expression de l'interdépendance des compétences des pouvoirs exécutif et législatif.}

Si la ratification ou l'approbation des traités et accords relatifs au domaine réglementaire s'effectue souverainement par le Chef de l'Etat ou le Gouvernement, il en va différemment de ceux portant sur le domaine matériel de la loi. Ceux-ci constituent le moment par excellence d'expression de l'interdépendance des pouvoirs exécutif et législatif. Alors que la ratification ou l'approbation de la convention internationale concernée est subordonnée à une autorisation parlementaire (a), l'opportunité de son exercice reste in fine soumise à l'appréciation discrétionnaire du Président de la République (b). 


\section{a. La subordination de la ratification/approbation des traités internationaux à une autorisation parlementaire.}

A rebours de la Constitution du Gabon (article 113), qui impose le « vote d'une loi d'autorisation » pour tous « les traités et les accords internationaux », sans considérations de leurs contenus et de leurs objets, les textes constitutionnels des autres Etats d'Afrique francophone subsaharienne ne prévoient, dans leur grande majorité, cette obligation que pour ceux relatifs au domaine matériel de la loi. Les Constitutions du Bénin (article 145), du Burkina-Faso (article 149), de la République Centrafricaine (article 69), de la République du Congo (article 178), de la République de Côte d'ivoire (article 120), de la République Démocratique du Congo (article 214), de la Guinée Conakry (article 149), du Mali (article 115), du Niger (article 169), du Sénégal (article 77), du Tchad (article 220), du Togo (article 139), exige, dans ce sens, l'adoption d'une loi d'autorisation parlementaire avant la ratification ou l'approbation de tout traité ou accord susceptible de modifier, de déroger, voire d'abroger les dispositions de nature législative. S'il est constant que cette loi d'autorisation ne saurait faire illusion, quant à l'étendue des compétences parlementaires sur la procédure de conclusion des traités à ratifier, il n'en demeure pas moins vrai qu'elle aiguille le comportement du Chef de l'Etat et celui du Gouvernement.

Sous ce rapport, la loi « visant à la ratification d'un traité ne saurait se confondre avec une loi ordinaire » (Blumann, 2007, p. 1044) : elle « (...) n'a de loi que le nom » (Rousseau, 1970, p. 108). La nature juridique de cette loi d'autorisation, qui est une « loi formelle » (Gicquel et Gicquel, 2017, p. 812 ; Drago, 2008, p 161), impose de ne pas la soumettre à un régime juridique identique à celui des autres procédures législatives. En outre, la loi autorisant le pouvoir exécutif à exprimer son consentement définitif à être lié, ne saurait être regardée comme un acte ordinaire «délibéré par l'Assemblée Nationale et le Sénat et promulguée par le Président de la République » (Avril et Gicquel, 2004, p. 152). En effet, les lois d'autorisation «n’édictent pas des règles de fond, mais constituent des décisions qui permettent au pouvoir exécutif d'accomplir valablement certains actes »(Avril et Gicquel, 2004, p. 152). Leur objet se résumerait ainsi à l'autorisation et non à la (ré)négociation des traités et accords internationaux. S'il est nécessaire aux parlementaires d'examiner le contenu de l'accord ou du traité faisant l'objet d'une demande d'autorisation, il ne leur est cependant pas autorisé d'y apporter des modifications au texte (Cottrel, 1983, p. 970 ; Maus, 1978, p. 1087), encore moins des réserves (Rambaud, 1977, p. 654). Produit de la volonté commune de plusieurs sujets de droit international, le traité ou l'accord ne saurait être modifié uniquement par la volonté unilatérale du Parlement d'un des Etats parties. L'hypothèse contraire consisterait à remettre en cause l'accord de volontés commun des sujets de droit international. 
En la matière, les Parlements des Etats d'Afrique francophone disposent «davantage d'une faculté d'empêcher que de la possibilité d'orienter» (Sur, 2011, p. 194) les traités ou accords nécessitant une autorisation d'approbation ou de ratification. Les organes du pouvoir exécutif se trouvent dans l'hypothèse du refus de l'assentiment des chambres tenus de surseoir à la procédure de conclusion de la convention internationale en cause. Le pouvoir d'autorisation de ratification/approbation ne saurait, dans ce sens, être qualifié de «résiduel» (Tcheuwa, 1997, p. 99), mais bien d'indispensable. Inversement, les premiers ne sont pas contraints de ratifier les traités et accords internationaux ayant reçu une onction parlementaire. L'exercice de la compétence de ratification/approbation qui dans la première hypothèse est dépendante de la volonté du Parlement, se trouve discrétionnaire dans la seconde hypothèse. Cette appréciation discrétionnaire de l'opportunité de ratification ou d'approbation rend, en retour, dépendante la volonté exprimée des parlementaires de voir le traité ou l'accord conclu, et partant, inséré dans l'ordre juridique national, de l'humeur du Président de la République ou du Gouvernement.

\section{b. L'appréciation discrétionnaire de l'opportunité de ratification/approbation du pouvoir exécutif.}

L'autorisation parlementaire préalable de ratification/approbation des traités internationaux, à l'issue d'un vote identique, a valeur d'avis sui generis : un avis ambivalent. Si l'introduction d'une demande d'autorisation de ratifier au Parlement est obligatoire, l'assentiment accordé à cette occasion ne lie pas l'autorité chargée de ratifier/approuver le traité ou l'accord en cause : le Chef de l'Etat ou le Gouvernement. Elle constitue tout simplement un acte formel préalable et indispensable à l'expression du consentement à être lié. A la suite de l'autorisation législative, le Président de la République ou le Gouvernement « retrouve la maîtrise totale de la prérogative d'engager l'Etat » (Kombi, 1996, p. 139) : une marge de discrétion dans l'exercice de sa compétence de ratifier/approuver. Il peut, en effet, ratifier immédiatement ou renvoyer son exercice à une date ultérieure. Une fois cette condition procédurale remplie, le Chef de l'Etat ou le Gouvernement devient le seul juge de l'opportunité et du moment de la ratification/approbation du traité ou de l'accord international. En d'autres termes, l'autorisation parlementaire « n'a pas une incidence politiquement impérative ou juridiquement contraignante » (Kombi, 1996, p. 139) à l'égard des organes du pouvoir exécutif.

L'alternative, de ratifier ou non, offerte au Président de la République et au Gouvernement ne saurait conduire à inférer une prééminence du pouvoir exécutif sur le pouvoir législatif dans la procédure de ratification des engagements internationaux en rapport avec le domaine matériel de la loi. Le pouvoir discrétionnaire laissé au premier après autorisation de ratification, du 
second, a, à quelques différences près, son pendant dans la procédure de formation des normes législatives. En effet, après le vote d'une loi par le Parlement, le Président de la République dispose d'une certaine marge de manœuvre dans sa promulgation (article 57 alinéa 2 de la Constitution du Benin ; article 31 alinéa 1, de la Constitution du Cameroun ; article 136 de la Constitution de la République Démocratique du Congo). La complexité des relations internationales justifie, semble-t-il, que le pouvoir discrétionnaire de ratifier/approuver du pouvoir exécutif ne soit pas enfermé dans des délais précis.

Sans considération de «l'importance de l'engagement international » (Turp, 2016, p. 18) en cause, le domaine matériel de la loi constitue le seul critère fondant l'intervention $\mathrm{du}$ Parlement en matière de ratification/approbation des traités ou accords internationaux. L'association du Parlement à cette procédure participe ainsi de la mise en cohérence de ses compétences normatives nationales avec celles internationales. Il est par-là réalisé une symétrie parfaite entre les premières et les secondes. A l'analyse, ce choix des constituants des Etats d'Afrique francophone fait surtout écho au « souci de protection de la compétence du Parlement (...) contre les empiètements que l'exécutif pourrait être tenté de réaliser par voie de traité » (Ascencio, 2012, p. 691 ; De Visscher, 1952, p. 556). Formulée en d'autres termes, cette solution constitue le point d'équilibre, entre les pouvoirs exécutif et législatif, dans la conduite des relations extérieures des Etats africains de succession française ou belge. Un tel équilibre trouvé, entre les pouvoirs, ne saurait en toute logique aller sans protection juridictionnelle.

\section{B. Un équilibre protégé, le contrôle juridictionnel de l'observance des compétences respectives des pouvoirs exécutif et législatif.}

L'équilibre des pouvoirs trouvé par les constituants africains, en maintenant intacts les périmètres respectifs des compétences du Parlement et de l'exécutif, au moment de l'expression du consentement définitif à être lié est a posteriori escorté par une protection juridictionnelle. En enjoignant, en effet, au juge de n'accorder « une autorité supérieure à celle des lois « qu'aux traités régulièrement approuvés » ou « ratifiés », ces prescriptions constitutionnelles le font apparaître « tel un équilibriste contraint de ménager la marge de manœuvre de l'exécutif tout en évitant de déposséder les représentants du peuple » (Bugorgue-Larsen, 2009, p. 1323). A l'occasion de cette mission, le juge de fond opère les contrôles du respect de la compétence d'autorisation du Parlement (1) et celui de la ratification/approbation par l'exécutif (2) avant d'appliquer un traité international au cours d'un procès. 


\section{Le contrôle du respect de la compétence d'autorisation du pouvoir législatif.}

Les Constitutions du Bénin (article 147), du Gabon (article 114), du Niger (article 171), de la République Centrafricaine (article 94), de la République Démocratique du Congo (article 215), du Rwanda (article 190), du Sénégal (article 77), du Tchad (article 220), du Togo (article 140), disposent de manière classique que : seuls les traités ou accords régulièrement ratifiés ou approuvés ont, dès leur publication, une autorité supérieure à celle des lois. Cet état du droit «constitutionnel africain commun» (Sindjoun, 1998) commande aux organes d'application des traités dans les ordres juridiques nationaux, et aux juges en particulier, de vérifier la régularité de ces textes insérés avant toute application.

A l'opposé de la Cour Constitutionnelle du Sénégal qui, depuis le 21 juin 2011 (Décision DCC 11-042 du 21 juin 2011, Ingrid Houessou, cons. 10), semble avoir accepté d'effectuer le contrôle de la conventionalité, et partant celui de la régularité des traités, les juridictions constitutionnelles des autres Etats africains reproduisent, pour l'heure, la solution française (Cons. Const., $\mathrm{n}^{0}$ 74-54 DC 15 janvier 1975, Interruption Volontaire de Grossesse). Il incombe donc au juge ordinaire, statuant au contentieux, et au juge constitutionnel en matière électoral en général, de vérifier le respect de la compétence d'autorisation du Parlement conformément au vœu des constituants africains.

Cependant, s'il est un domaine où le juge « s'immisce à contrecœur » (Pellet et Miron, 2015, p. 395), le contrôle du respect des compétences du Parlement dans la procédure de conclusion des traités, est bien celui-là. Le juge ne dispose pas toujours d'un critère, facile à manier, pour identifier le périmètre au-delà duquel l'approbation en forme législative n'était plus indispensable, de celui dans lequel celle-ci était fondamentale. La porosité (Le Bœuf, 2016, p. 614) des catégories énumérées par la plupart des Constitutions africaines rend, en effet, peu aisée l'office du juge dans ce contrôle du respect des compétences d'autorisation d'un traité ou d'un accord international au cours de sa conclusion. La solution, certes isolée, de l'article 43 de la Constitution camerounaise, consistant à faire coüncider la compétence d'autorisation des représentants du peuple avec le domaine matériel de la loi n'est, dans ce sens, pas sans pertinence. Toutefois, ce contrôle de la régularité de l'approbation se révèle variable suivant que le Parlement ait adopté une loi d'autorisation, ou non.

Le contrôle du respect de la compétence du pouvoir législatif ne présente, sur un plan purement théorique, pas de grande difficulté dans le cas d'une absence d'autorisation préalable des parlementaires. L'absence d'une autorisation en forme législative préalablement à la ratification/approbation d'un traité ou d'un accord international constitue une violation évidente de 
l'équilibre des pouvoirs, qu'il appartient aux juges de sanctionner. Ceux-ci étant en vertu de la disposition classique et commune aux Constitutions africaines, habilités à faire prévaloir un engagement international sur une loi, ils le sont «tout autant pour vérifier préalablement si les conditions de l'applicabilité » (Raynaud et Fombeur, 1999, p. 127) du premier ont été bien remplies.

A l'inverse, l'intervention effective du Parlement dans la procédure de conclusion des traités rend en général fort complexe, voire impossible, le contrôle juridictionnel de sa régularité. Deux raisons semblent être au fondement de cette complexité. La première, est l'incompétence du juge ordinaire pour connaitre de la procédure de fabrication des lois. Seul le Conseil Constitutionnel est apte à juger, et ce a priori, de la régularité (Magnon, 2008, p. 85) de la procédure législative. Une fois la loi d'approbation adoptée, la régularité de sa formation devient indiscutable devant le juge : « le contrôle a posteriori sur les lois, ne concerne jamais leur procédure d'adoption » (GoeselLe Bihan, 1995, p. 64).

Bien mieux, et c'est le second argument, la théorie de la loi écran fait, obstacle à l'intervention du juge ordinaire dans le contrôle du respect des compétences du pouvoir législatif. Dans cette mesure, le juge devant qui est invoqué un traité ou un accord international ayant été approuvé est incompétent à apprécier la validité de sa procédure d'autorisation. La loi autorisant la ratification fait, en effet, écran (Donnat et Casas, 2002, p. 1005) à tout contrôle juridictionnel éventuel. Le Conseil d'Etat français a, dans ce sens, jugé qu'«il ne lui appartient pas, en revanche, dès lors que (...) l'approbation est intervenue en vertu d'une loi, de se prononcer sur le moyen tiré de ce que la loi autorisant cette ratification (...) serait contraire à la Constitution » (CE Sect., 8 juillet 2002, n 239366, Commune de Porta). Les juges ordinaires africains se positionnent davantage comme des vigiles des compétences du Parlement en matière d'autorisation de ratification/approbation des traités, que comme des censeurs de leur légalité. Ceci sans préjudice de la compétence de ratification du pouvoir exécutif.

\section{Le contrôle du respect de la compétence de ratification/approbation du pouvoir exécutif.}

L'exigence constitutionnelle selon laquelle, « les traités ou accords internationaux régulièrement (...) ratifiés ont, dès leur publication, une autorité supérieure à celles des lois », pose deux exigences au juge de fond au moment de son application : il doit se montrer « ouvert au contrôle et à la garantie de la normativité internationale »(Taxil, 2011, p. 417). Le juge interne étant sans compétence pour vérifier la validité internationale (CE, 5 février 1926, Dame Caraco) d'un traité ou d'un accord international, il lui appartient cependant de s'assurer de la régularité de la conclusion de ce dernier 
au regard des règles nationales en vigueur. Au nombre de celles-ci, figure la condition de ratification, dont la réalisation, dans certains cas, en l'absence d'une loi d'autorisation ne saurait être regardée comme régulière (CE ass., 18 décembre 1998, $\mathrm{n}^{0}$ 181249, SARL du parc d'activités de Blotzheim). Ce contrôle de la « régularité de la ratification constitue nécessairement l'une des conditions posées à la supériorité des traités sur les lois dans la hiérarchie des normes » (Pellet et Miron, 2015, p. 297) qu'il incombe in fine au juge de vérifier.

La conduite des relations internationales, et la conclusion des traités internationaux en particulier, constituant le lieu où la théorie des actes de Gouvernement, ou mieux des actes «injustifiables» (Melleray, 2007, p. 1320 ; Favoreu, 2003, p. 610), joue en déployant toute sa vigueur (Serrand, 2003, p. 15), les juges africains ne s'y aventurent qu'avec beaucoup de prudence dans l'appréciation de la condition de ratification. Le contrôle de la régularité du décret de ratification/approbation d'un traité ou d'un accord international se retranche ainsi d'ordinaire dans celui de la vérification de la compétence de son signataire.

C'est du moins ce que laisse inférer la motivation d'une décision $\mathrm{du}$ juge judiciaire camerounais dans l'application de la Convention sur l'Elimination de toutes les Formes de Discrimination à l'Egard des Femmes (CEDEF). Dans un de ses considérants, le juge précise que : «l'Etat du Cameroun a régulièrement ratifié la CEDEF du 15 juillet 1988 et cette convention est entrée en vigueur le 23 aout 1994 »(TPI/YCA, Ord., n 457/C 14 mars 2005, affaire Ngoa née Sollo Mireille c/ Ngoa Kisito). Afin d'écarter les articles 21, 371 et 374 du Code Civil, en cause dans cette affaire, le juge a implicitement vérifié la compétence de l'auteur du décret de ratification de ladite convention. Le contrôle «minimum » du décret de ratification, plus exactement de la compétence du Président de la République à ratifier la CEDEF, a servi au juge civil, à faire jouer pleinement l'article 45 de la Constitution pour écarter les dispositions du Code Civil. Dans une décision similaire, le Tribunal de Grande Instance du département du Mfoundi (Cameroun) a fondé sa décision en ces termes : « (...) que l'article 45 de la loi n 96/06 du 18 janvier 1996 portant révision de la Constitution de 1972, énonce que les traités et accords internationaux régulièrement approuvés ou ratifiés ont, dès leur promulgation, une autorité supérieure à celle des lois internes ; que l'article 16 de la CEDEF affirme que l'épouse a les mêmes responsabilités que l'époux (...) » (TGI du Mfoundi, jugement civil, n $420 \mathrm{du}$ 21 mai 2008, affaire Dame Ndingue Anne épouse Tchoumba c/ Ngomsi Kamguia Tchoumbé Dieudonné). Ce contrôle de l'existence d'un décret de ratification, du Président de la République paraît d'ailleurs plus marqué dans l'affaire Ministère public et administration des douanes contre Kamté et autres. Le juge de la Cour d'Appel du Littoral a, en l'espèce, précisé que : le 
tarif des douanes de l'UDEAC, contesté « (...) est régulièrement ratifié par le décret $n^{\circ}$ 65/DF/572 du 31 décembre 1965 en son paragraphe II de l'article premier et publié au journal officiel de la R.F.C., n' 1 du $1^{\text {er }}$ janvier $1966 »$ (CA/LI, arrêt, n ${ }^{\circ}$ 10006/P du 20 juillet 1982, Ministère public et administration des douanes c/ Kamté Honoré et autres). Il apparaît bien, dans cette dynamique, que le juge ordinaire protège concomitamment, les compétences des pouvoirs législatif et exécutif (Ondoua, 2014, p. 299).

\section{Conclusion}

L'idée forgée au sein de la doctrine suivant laquelle «toutes les Constitutions modernes consacrent la primauté de l'exécutif en matière de politique étrangère » (Zoller, 1992, p. 31), s'avère d'une pertinence partielle en Afrique francophone. Le déséquilibre observable dans l'attribution et la délimitation de la compétence de négociation ne saurait suffire à inférer le règne d'un «présidentialisme outrancier » (Sindjoun, 1993, p. 814) dans la conclusion des traités, sauf à ensevelir celle-ci « sous le voile des mystères de la diplomatie » (Goesel Le-Bihan, 1995, p. 9). La montée en puissance du Chef de l'Etat, dans la majorité des Etats africains francophones, favorisée par « l'autonomie, la plénitude, la généralité et l'exclusivité » de son pouvoir en matière de négociation des conventions internationales (Kombi, 2003, p. 10) est sans une incidence significative sur les compétences traditionnelles du pouvoir législatif. L'examen de l'articulation de la procédure de ratification/approbation, qui apparaît comme un « bon indice révélant la place respective des pouvoirs constitutionnels »(Pellet et Miron, 2015, p. 292), donne à constater un équilibre certain entre ces derniers. Aucun des organes des pouvoirs exécutif et législatif ne se trouve dépouillé de ses attributions constitutionnelles au visa de la conclusion d'un traité ou d'un accord international. L'équilibre des pouvoirs manqué dans l'organisation de la compétence de négociation des traités internationaux est ainsi trouvé dans l'articulation constitutionnelle de l'expression du consentement définitif à y être lié. Le baobab présidentiel ne saurait donc plus cacher la forêt parlementaire dont le pouvoir d'autorisation, n'est pas seulement « symbolique » (Bugorgue-Larsen, 2009, p. 1316) mais, reste central en matière de ratification/approbation d'une catégorie d'engagements internationaux bien déterminés.

\section{References:}

1. Ascencio, H. (2012). «Les relations extérieures », in Troper, M. et Chagnollaud, D. Traité international de droit constitutionnel, Tome II, Paris, Dalloz.

2. Atangana Amougou, J-L. (2006). «La négociation dans l'exécution des arrêts de la Cour Internationale de Justice », R.R.J-D.P., n ${ }^{0} 3$. 
3. Avril, P. et Gicquel J. (2004). Droit parlementaire, $3^{\mathrm{e}}$ éd., Paris, Montchrestien.

4. Barberis, M. (2012). «La séparation des pouvoirs », in Troper, M. et Chagnollaud D. Traité international de droit constitutionnel, Tome I, Paris, Dalloz.

5. Basdevant, J. (1926). «La conclusion et la rédaction des traités et instruments diplomatiques autres que les traités », R.C.A.D.I., Tome 15.

6. Basdevant, J. (1963) Dictionnaire de la terminologie du droit international, Paris, Sirey.

7. Blumann, C. (2007). «Un mimétisme à l' envers : le contrôle de constitutionnalité des engagements externes en droit constitutionnel et communautaire », in Renouveau du droit constitutionnel, Mélanges en l'honneur de Louis Favoreu, Paris, Dalloz..

8. Bugorgue-Larsen, L. (2009). « Article 53 », Luchaire F. Conac, G. et Pretot, X. La Constitution de la République Française. Analyses et commentaires, Paris, Economica.

9. Cabanis, A. et Martin M-L. (2010). Le constitutionnalisme de la troisième vague en Afrique francophone, Bruxelles, Bruylant.

10. Cottrel, Y. (1983). « Note sur le contrôle du Parlement sur la pratique étrangère de la France sous la $\mathrm{V}^{\mathrm{e}}$ République », R.D.P.

11. De Villers, M. et Le Divellec, A. (2017). Dictionnaire du droit constitutionnel, $11^{\mathrm{e}}$ éd., Paris, Sirey.

12. De Visscher, P. (1952). «Les tendances internationales des Constitutions modernes », R.C.A.D.I., vol. 80.

13. DESOUCHES, C. (1979) «Les parlements », in Conac, G. (dir.), Les institutions constitutionnelles des Etats d'Afrique francophone et de la République Malgache, Paris, Economica.

14. Donnat, F. et Casas, D. (2002). «Les limites du contrôle du juge administratif sur l'acte de publication d'un engagement international dont la ratification a été autorisée en vertu d'une loi », A.J.D.A.

15. Drago, G. (2008). «Le Parlement et les traités internationaux. Considérations sur l'autorisation parlementaire de ratification des engagements internationaux », in Constitutions et pouvoirs, Mélanges en l'honneur de Jean Gicquel, Paris, Montchrestien.

16. Du Bois de Gaudusson, J. (2008). «Sur l'attractivité du modèle de la Constitution de 1958 en Afrique, cinquante ans après... », in Mathieu, B. (2008). (dir.), 1958-2008: Cinquantenaire de la Constitution française, Paris, Dalloz.

17. Duguit, L. (1924). Traité de droit constitutionnel, $2^{\mathrm{e}}$ éd., Paris. 
18. Favoreu, L. (2003). «Pour en finir avec la 'théorie', des actes de Gouvernement », in L'esprit des institutions, l'équilibre des pouvoirs, Mélanges en l'honneur de Pierre PACTET, Paris, Dalloz.

19. Gaia, P. (1991). Le Conseil constitutionnel et l'insertion des engagements internationaux dans l'ordre juridique interne, Thèse publiée, Paris, Economica-PUAM.

20. Geamanu, G. «Théorie et pratique des négociations en droit international », R.C.A.D.I., T. 166, 1980.

21. Gicquel, J. et Gicquel, J-E. (2017). Droit constitutionnel et institutions politiques, Paris, 30 éd., L.G.D.J.

22. Goesel-Le Bihan V. (1995). La répartition des compétences en matière de conclusion des accords internationaux sous la $\mathrm{V}^{\mathrm{e}}$ République, Thèse publiée Paris, Pédone.

23. Goesel-Le Bihan, V. (1997). «Sur quelques aspects récents du droit constitutionnel français des relations extérieures », A.F.D.I., Vol. 43.

24. Gonidec, P-F. (1982). «Le droit international », in Encyclopédie de l'Afrique, Tome. I : L'Etat de droit.

25. Isidoro, C. (2009). «Article 52 », in Luchaire, F. Conac, G. et Pretot, X. La Constitution de la République Française. Analyses et commentaires, Paris, Economica.

26. Kante, B. (2011). «La production d'un nouveau constitutionnalisme en Afrique: Internationalisation et régionalisation $\mathrm{du}$ droit constitutionnel », in Land, law, and politics in Africa, Mélanges en mémoire de Gerti Hesseling, Brill.

27. Kontchou Kouomegni, A. (1979). «Les constitutions africaines et le droit international », in Conac, G. (dir.), Les institutions constitutionnelles des Etats d'Afrique francophone et la République malgache, Paris, Economica.

28. Le Bœuf, R. (2016). « La double nature de la ratification des traités : observation sur les discordances entre les procédures constitutionnelles et internationales », R.F.D.C., $\mathrm{n}^{0} 3$.

29. Locke, J. (1953) Essai sur le gouvernement civil, Paris, P.U.F.

30. Magnon, X. (2008). Théorie(s) du droit, Paris, Ellipses, coll. Universités.

31. Manin, P. (1979). «Article 52 », in Luchaire, F. et Conac, G. La Constitution de la République française, Paris, Economisa.

32. Maus, D. (1978). «L'Assemblée Nationale et la ratification des traités », R.D.P.

33. Melleray, F. (2007). «En a-t-on fini avec la théorie des actes de gouvernements ? », in Renouveau du droit constitutionnel, Mélanges en l'honneur de Louis Favoreu, Paris, Dalloz. 
34. Metou, B-M. (2009). « Le moyen de droit international devant les juridictions internes en Afrique : quelques exemples d'Afrique noire francophone », R.Q.D.I.,

35. Montesquieu. (1964). De l'Esprit des lois, Paris, Le seuil,

36. Mouelle Kombi, N. (1992). Les compétences internationales du Cameroun. Contribution à l'étude de l'action internationale d'un Etat du tiers monde, T. I, Thèse de doctorat, Université René Descartes Paris V.

37. Mouelle Kombi, N. (1996). «La loi constitutionnelle du 18 janvier 1996 et le droit international », Melone, S., Minkoa She, A. et Sindjoun. La réforme constitutionnelle du 18 janvier 1996. Aspects juridiques et politiques, GRAP/Fondation Ebert, Yaoundé.

38. Mouelle Kombi, N. (1996). La politique étrangère du Cameroun, Paris, L'Harmattan, coll. « Points de vue ».

39. Mouelle Kombi, N. (2003). " Les dispositions relatives aux conventions internationales dans les nouvelles constitutions des Etats d'Afrique francophones », R.J.P.I.C., 2003, $\mathrm{n}^{\mathrm{o}} 1$.

40. Nourou Tall, S. (2017). «Les références au droit international public dans les Constitutions africaines francophones : exemples du Benin, du Sénégal et du Togo », Démocratie en questions, Mélanges en l'honneur du professeur Theodore Holo, Paris, P.U.T.

41. Olinga A-D. (2013). La constitution de la République du Cameroun, $2^{\mathrm{e}}$ éd., Yaoundé, PUCAC.

42. Ondoua, A. (2014). «L'internationalisation des Constitutions en Afrique subsaharienne et la protection des droits fondamentaux », R.T.D.H., $\mathrm{n}^{\circ} 98$.

43. Ondoua, A. (2014). «Le droit international dans la constitution camerounaise », in Atangana Amougou J-L. (dir.), Le Cameroun et le droit international colloque de Ngaoundere, Paris, Pedone.

44. Ondoua, A. (2017). « La Constitution béninoise du 11 décembre 1990 et les normes d'origine externe », in Démocratie en questions, Mélanges en l'honneur du professeur Theodore Holo, Paris, P.U.T.

45. Pactet, P. et Melin-Soucramanien, F. (2014). Droit constitutionnel, $33^{\mathrm{e}}$ éd., Paris Dalloz.

46. Rambaud, P. (1977). « Le Parlement et les engagements internationaux de la France sous la V ${ }^{\mathrm{e}}$ République », R.G.D.I.P.

47. Raynaud, F. et Fombeur, P. (1999) « Conditions d'application en France d'un traité dont la ratification ou l'approbation est intervenue sans avoir été autorisée par une loi », A.J.D.A., 1999.

48. Rousseau, C. (1970). Droit international public, introduction et sources, Tome I, Paris, Sirey.

49. Saidj, L. (1979). Le Parlement et les traités. La loi relative à la 
ratification ou à l'approbation des engagements internationaux, Paris, LGDJ.

50. Sall, A. (1997). « Le droit international dans les nouvelles constitutions africaines », R.J.P.I.C.

51. SALMON, J. (2001), (dir.) Dictionnaire de droit international public, Bruxelles, Bruylant/AUF.

52. Serrand, P. (2003). « Acte de gouvernement », in Alland. D. et Rials, S. (dir.), Dictionnaire de la culture juridique, Paris, P.U.F., coll. Quadridge.

53. Simon, D. (1995). « Préface », in Goesel-Le Bihan, V. La répartition des compétences en matière de conclusion des accords internationaux sous la V $\mathrm{V}^{\mathrm{e}}$ République, Thèse publiée, Paris, Pedone.

54. Sindjoun, L. (1993). «L'action internationale de l'Assemblée Nationale du Cameroun éléments d'analyste politiste », Etudes Internationales, vol. $24, n^{0} 4$.

55. Sur, S. (2011). Relations internationales, $6^{\mathrm{e}}$ éd., Paris, Montchrestien.

56. Taxil, B. (2011). « Normes internationales », in Gonod, P., Melleray, F. et Yolka, P. (dir.), Traité de droit administratif, T. I, Paris, Dalloz.

57. Tcheuwa, J-L. (1997). «Quelques aspects du droit international à travers la nouvelle constitution camerounaise du 18 janvier 1996 », RJPIC.

58. Tourard, H. (2000). L'internationalisation des Constitutions nationales, Thèse publiée, Paris, L.G.D.J., coll. «Bibliothèque constitutionnelle et de science politique ».

59. Troper, M. (1998). «Les relations extérieures dans la constitution de l'an III. Vers la fonction gouvernementale», in, L'évolution du droit international, Mélanges offerts à Hubert Thierry, Paris, Pedone.

60. Turp, D. (2016). «L'approbation des engagements internationaux importants au Québec: la nouvelle dimension parlementaire à la doctrine Gérin-Lajoie », R.Q.D.I., HS.

61. Vandendriessche, X. (2001). «Le parlement entre déclin et modernité », pouvoirs, $\mathrm{n}^{0} 99$.

62. Zoller, E. (1992). Droit des relations extérieures, Paris, P.U.F., coll. Droit fondamental, 1992. 